\title{
Percutaneous Vertebroplasty versus Conservative Treatment Using a Transdermal Fentanyl Patch for Osteoporotic Vertebral Compression Fractures
}

\author{
Younggyu Oh, ${ }^{1}$ Byungjou Lee, ${ }^{2}$ Subum Lee, Junghwan Kim, ${ }^{3}$ Jinhoon Park ${ }^{1}$ \\ Department of Neurological Surgery, Asan Medical Center, University of Ulsan College of Medicine, Seoul, Korea \\ Department of Neurosurgery, ${ }^{2}$ Inje Universiry Ilsan Paik Hospital, Neuroscience \& Radiosurgery hybrid Research Center, Inje Universiry \\ College of Medicine, Ilsan, Korea \\ Department of Orthopaedic Surgery, ${ }^{3}$ Gangneung Asan Hospital, University of Ulsan College of Medicine, Gangneung, Korea
}

Objective : Although surgical intervention, such as percutaneous vertebroplasty (PVP), is the standard treatment for osteoporotic vertebral compression fractures (OVCFs), its effectiveness and safety are unclear. Therefore, this study compared the safety and efficacy of conservative treatment with that of PVP for acute OVCFs.

Methods : Patients with single-level OVCFs who were treated conservatively with a transdermal fentanyl patch (TFP) or with PVP between March 2013 and December 2017 and followed-up for more than 1 year were retrospectively evaluated. Patients with pathologic fractures, fractures of more than two columns, or a history of PVP were excluded. Clinical outcomes (visual analog scale [VAS] scores) and radiographic factors were evaluated, including changes in the compression rate of the corresponding vertebral body at onset and after 12 months, sagittal Cobb angle at onset and after 6 and 12 months, and the incidence of adjacent compression fractures.

Results : Of the 131 patients evaluated, 75 were treated conservatively using TFPs and 56 underwent PVP. We divided the patients into TFP and PVP groups. Their baseline characteristics (including sex, level of fracture, and bone mineral density T-scores) were similar, but the TFP group was significantly younger. The overall VAS score for pain showed a greater decrease during the first month (1 week after PVP) in the PVP group but remained similar in the two groups thereafter. The compression rate after 12 months increased in the TFP group but decreased in the PVP group. Five patients in the PVP group, but none in the TFP group, experienced adjacent compression fractures within 12 months.

Conclusion : We compared clinical and radiological outcomes between the TFP and PVP groups. The immediate pain reduction effect was superior in the PVP group, but the final clinical outcome was similar. Although the PVP group had a better-preserved compression rate than the TFP group for 1 year, the development of adjacent fractures was significantly higher. Although TFPs seemed to be beneficial in reducing the failure rate of conservative treatment, the possibility of side effects $(22.6 \%$, 17 out of 75 patients, in this study) should be carefully monitored.

Key Words : Fractures, Compression · Vertebroplasty · Conservative treatment.

\footnotetext{
- Received : April 9, 2019 •Revised : June 16, 2019 •Accepted : June 28, 2019

- Address for reprints : Jinhoon Park

Department of Neurological Surgery, Asan Medical Center, University of Ulsan College of Medicine, 88 Olympic-ro 43 gil, Songpa-gu, Seoul 05505, Korea Tel : +82-2-3010-3550, Fax : +82-2-476-6738, E-mail : jhpark@amc.seoul.kr, ORCID : https://orcid.org/0000-0002-0903-3146
} 


\section{INTRODUCTION}

Vertebral compression fractures are the most common type of osteoporotic fractures, with approximately 1.4 million cases per year noted worldwide ${ }^{6}$. Vertebral compression fractures can result in severe pain, an inability to perform activities of daily living, and a marked reduction in quality of life ${ }^{24)}$. Moreover, these fractures are associated with increased age-adjusted mortality ${ }^{21}$. To reduce this burden, evidence-based prevention and management are essential ${ }^{1,20)}$.

Over the past few years, percutaneous vertebroplasty (PVP), a minimally invasive technique, has been widely used worldwide to treat painful osteoporotic vertebral compression fractures (OVCFs) ${ }^{9,19,25)}$. However, the effect of percutaneous cement augmentation are unclear, as are the benefits of PVP (including pain control, hospitalization period, quality of life, and vertebral body height restoration) and its adverse procedure-related events and incidence of adjacent compression fractures ${ }^{7,8,12-15,18,22,26)}$. Conservative treatment may also benefit patients; hence, comparing the effects of conservative and PVP treatments on clinical and radiologic outcomes is essen- tial. However, no concrete guidelines exist on how to administer conservative treatment in terms of medication, duration of bed rest, and use of orthoses.

This study compared the clinical and radiologic outcomes of PVP and conservative therapy using transdermal fentanyl patches (TFPs) in patients with OVCFs' ${ }^{2)}$.

\section{MATERIALS AND METHODS}

\section{Study design and patient groups}

The Institutional Review Board of Gangneung Asan Hospital approved this study (approval number : 201905005 GNAH IRB). This study included patients who were diagnosed with recent OVCFs and were treated conservatively using TFP or with PVP by two different spinal surgeons, according to the different treatment approaches, from March 2013 to December 2017.

Patients were included if they had single-level OVCFs, no history of treatment for vertebral fractures, no current medical comorbidity, and had been followed-up for at least 1 year

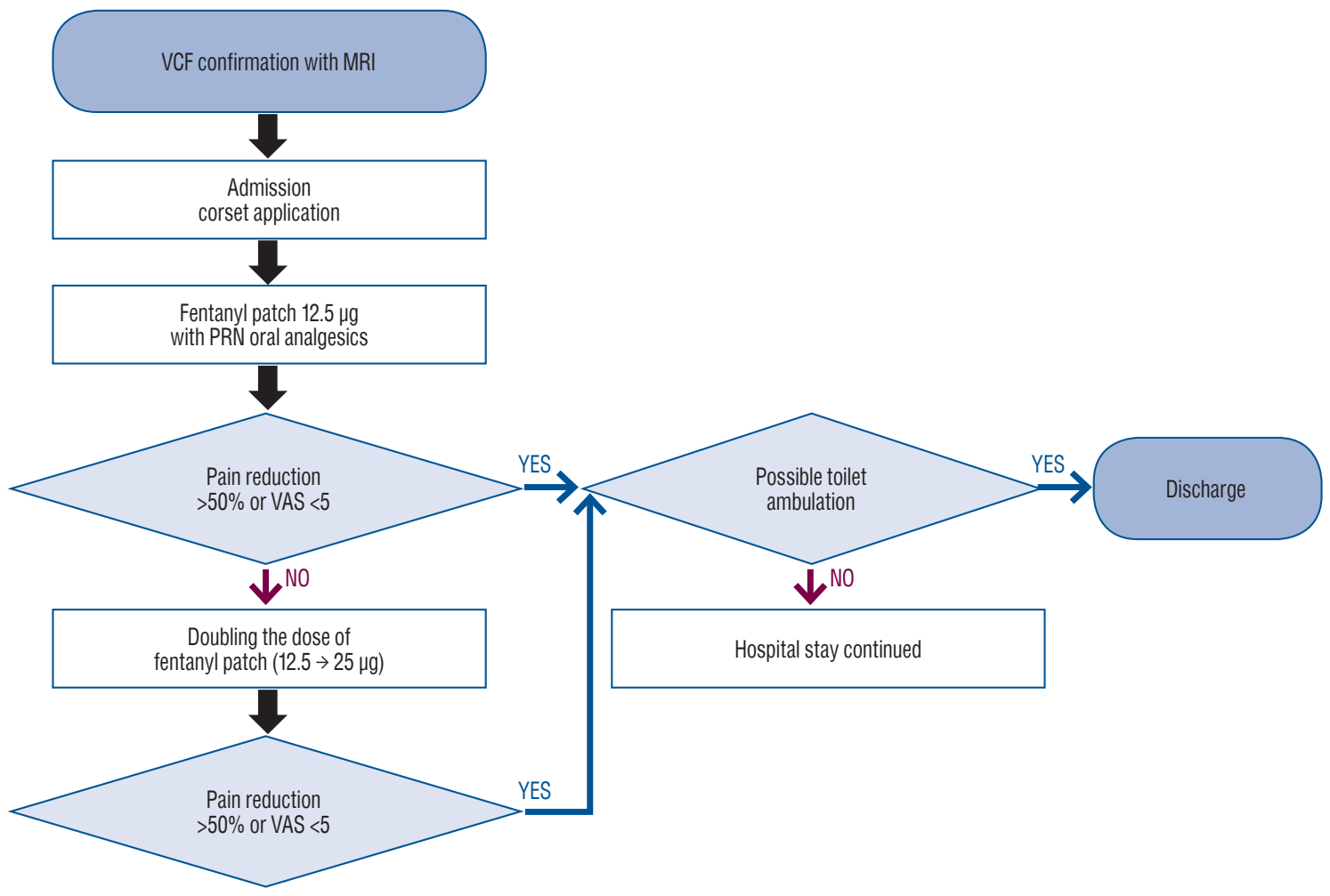

Fig. 1. Treatment protocol for the transdermal fentanyl patch group. VCF : vertebral compression fracture, MRI : magnetic resonance imaging, PRN : pro re nata, VAS : visual analog scale. 
with periodic evaluation of spine X-rays (anteroposterior/lateral views).

Patients were excluded if they had neurological deficits, pathological fractures, or unstable vertebral fractures involving the middle or posterior column of the spine.

Finally, 75 patients who underwent conservative treatment using TFPs and 56 patients who underwent PVP with a visual analog scale (VAS) score of 5 or more after 3 weeks of prevalent conservative treatment were included.

Statistical analysis included the student's t-test, MannWhitney $U$ test, and Fisher's exact test, and a $p$-value of less than 0.05 was regarded as significant.

\section{Treatment protocols for the conservative treat- ment and TFP groups}

All patients underwent magnetic resonance imaging and assessment of bone mineral density (BMD) at admission and began osteoporosis medication and wore a corset for at least 3 months. After admission, a low-dose $(12.5 \mu \mathrm{g})$ TFP was administered. Patients were prescribed combination tablets of tramadol (37.5 $\mathrm{mg}$ ) and acetaminophen $(325 \mathrm{mg})$ or acetaminophen (250 mg), ibuprofen (200 mg), and codeine phosphate $(10 \mathrm{mg})$ as pro re nata oral medications.

After the low-dose $(12.5 \mu \mathrm{g})$ fentanyl patch application, the patients were asked whether their pain VAS score had changed. If the VAS score had decreased to below 5 or was $50 \%$ below the initial score, we continued to use the low-dose fentanyl patch for 1 month. However, if the patient complained of sustained pain with a VAS score that was more than 5 or $50 \%$ above the initial score, we increased the dose of the fentanyl patch by $25 \mu \mathrm{g}$. The fentanyl patches and corsets were applied simultaneously and immediately after admission. All
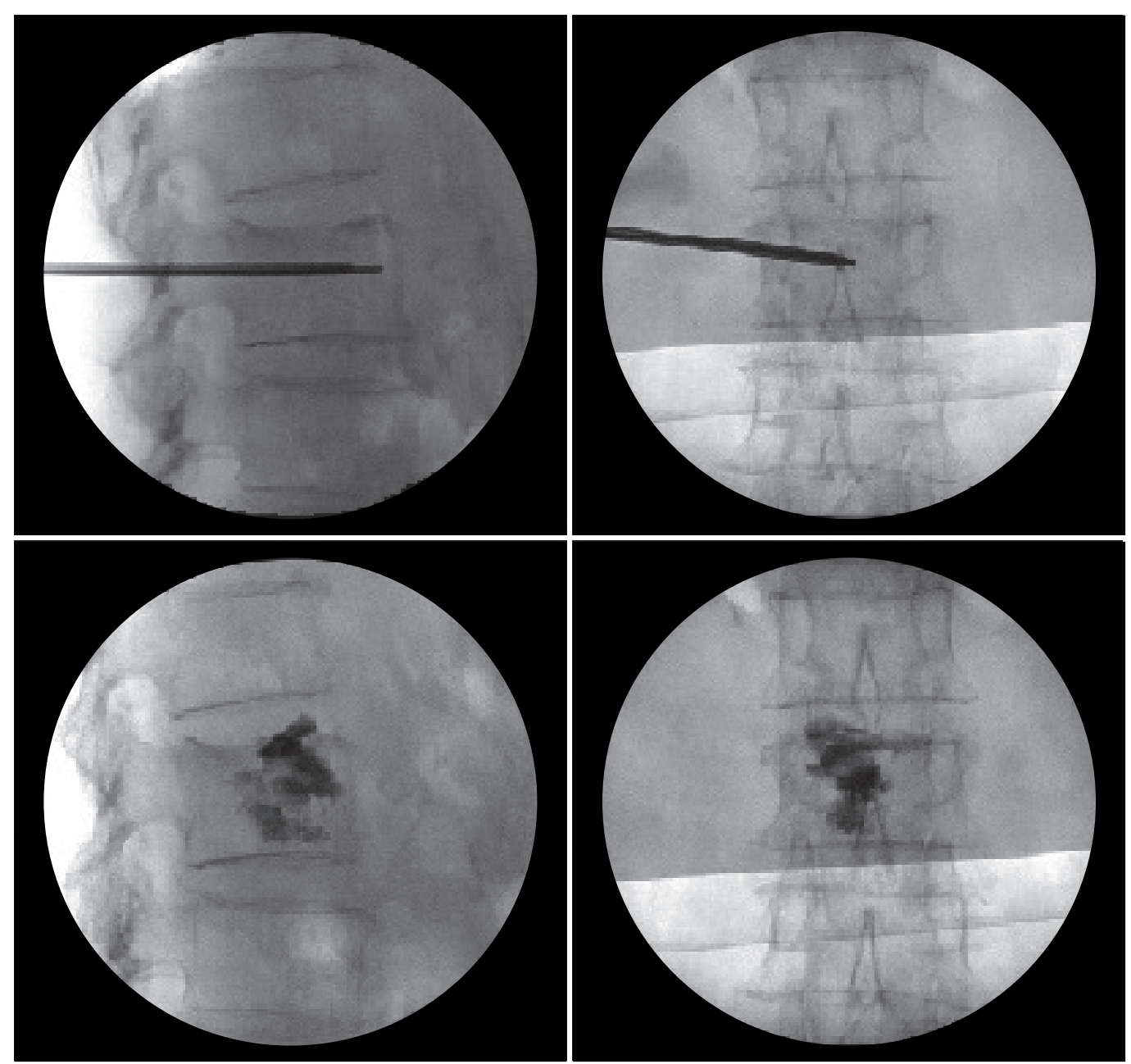

Fig. 2. Scans showing the percutaneous vertebroplasty (PVP) procedure. Following maximal postural reduction during PVP, high-viscosity cement was slowly injected at low pressure to maximize kyphosis reduction. 
patients were encouraged to use the toilet by walking with or without assistance, according to their pain tolerance. Absolute bed rest was not recommended, and discharge was recommended when toilet ambulation was possible and the VAS score was under 5 (Fig. 1).

\section{Treatment protocol for the PVP group}

In the PVP group, patients with sustained pain with a VAS score of more than 5 after 3 weeks of conservative treatment underwent PVP. Of the 170 patients with compression fractures, 56 (32.9\%) were included and underwent PVP, based on the aforementioned guideline. This conservative treatment was performed with a non-steroidal anti-inflammatory drugs, COX-2 inhibitors, or combination tablets (tramadol and acetaminophen). Early ambulation was also encouraged after a brace was worn after identifying pain tolerance.

A vertebroplasty needle was inserted using a unilateral transpedicular approach. The liquid and powder components of polymethylmethacrylate were mixed, and the cement was injected through the pedicle under continuous fluoroscopic monitoring in the lateral view, with close attention paid to the posterior margin of the vertebral body and the epidural space. Postural reduction was also performed during PVP (Fig. 2).

Patients were discharged from the hospital after they attained tolerable toilet ambulation.

\section{Comparison of baseline characteristics and pa- tient outcomes}

The two groups were compared for factors including age,

Table 1. Baseline demographic and clinical characteristics of the patients in the two groups

\begin{tabular}{lccc}
\hline & $\begin{array}{c}\text { TFP group } \\
(\mathbf{n}=75)\end{array}$ & $\begin{array}{c}\text { PVP group } \\
(\mathbf{n}=56)\end{array}$ & $p$-value \\
\hline Age (years) & $63.4 \pm 15.3$ & $71.5 \pm 8.1$ & 0.001 \\
Sex (M/F) & $28 / 47$ & $20 / 36$ & 0.849 \\
Level of fracture & & & 0.067 \\
$\quad$ Thoracic (T5-T10) & $2(2.7)$ & $6(10.7)$ & \\
Thoracolumbar (T11-L2) & $66(88.0)$ & $41(73.2)$ & \\
Lumbar (L3-L5) & $7(9.3)$ & $9(16.1)$ & \\
BMD T-score & $-2.8(1.0)$ & $-2.9(0.6)$ & 0.751 \\
\hline
\end{tabular}

Values are presented as mean \pm standard deviation or number (\%) unless otherwise indicated. TFP : transdermal fentanyl patch, PVP : percutaneous vertebroplasty, $\mathrm{M}$ : male, $\mathrm{F}$ : female, BMD : bone mineral density sex, fracture level, and T-score of BMD (Table 1). The back pain VAS score was evaluated and compared at onset as well as 3 weeks and 1, 3, 6, and 12 months after the injury.

Radiographs, including anteroposterior and lateral X-ray images, were taken and compared at onset and 6 and 12 months after the injury. The compression rate was determined and compared by measuring the ratio of the anterior and posterior heights of the fractured vertebra level. The vertebral body wedge angle (kyphotic angle) was determined and compared by measuring the angle between the superior endplate of the vertebral body above and the inferior endplate of the vertebral body below the fractured vertebra on lateral X-rays (Fig. 3). The development of compression fractures adjacent to the index vertebra during follow-up was checked and compared.

\section{RESULTS}

The patients in TFP group were carefully observed for side effects of the fentanyl patch, such as nausea, vomiting, or dizziness. Such side effects developed in 17 patients (22.6\%), even after the use of a low-dose $(12.5 \mu \mathrm{g})$ fentanyl patch. We subsequently removed the patch from their medication and instead

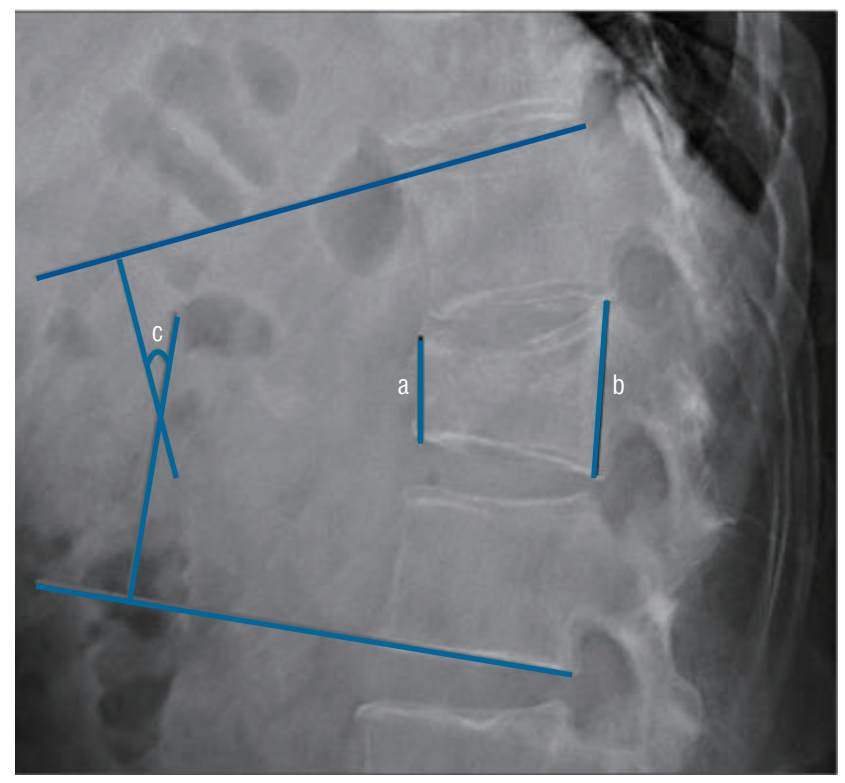

Fig. 3. X-ray showing the method used to calculate the compression rate. This rate was calculated as $(b-a) / b \times 100$, where a represents the anterior vertebral height and $\mathrm{b}$ represents the posterior vertebral height. The Cobb angle is measured as $\mathrm{c}$. 
administered only oral analgesics.

In 16 patients $(21.3 \%)$ whose pain was not controlled with the low-dose $(12.5 \mu \mathrm{g})$ fentanyl patch, a higher dose of fentanyl $(25 \mu \mathrm{g})$ was used. Six of the 75 patients underwent PVP because of insufficient pain reduction and low overall satisfaction.

\section{Comparison of clinical outcomes between the TFP and PVP groups}

During the 12-month follow-up period, overall VAS scores improved over time in both groups (from 7.8 to 1.3 in the TFP group, and from 7.3 to 1.5 in the PVP group). However, no statistical difference was observed between the two groups when changes were compared from the baseline to 12 months (6.5 in the TFP group, 5.8 in the PVP group, $p=0.62$ ). The mean VAS scores of the TFP and PVP groups were 5.6 and 6.1, respectively, at 3 weeks $(p=0.355)$. However, a significant difference in the VAS score was observed between the two groups at 4 weeks ( 1 week after PVP) (VAS score, 5.8 in the TFP group; VAS score, 3.4 in the PVP group; $p=0.022$ ) (Fig. 4).

\section{Comparison of radiologic outcomes between the TFP and PVP groups}

The compression rates in the TFP and PVP groups were 24.8 and 32.9, respectively, at admission. The baseline compression rate was significantly higher in the PVP group ( $p=0.001$ ). After 12 months, the compression rates of the TFP and PVP groups were 31.9 and 28.1, respectively. Although kyphosis progressed in the TFP group, compression did not progress after kyphosis reduction in the PVP group $(p=0.001)$.

The mean kyphotic angles in the TFP and PVP groups were $13.2^{\circ}$ and $14.8^{\circ}$, respectively, at baseline $(p=0.002)$. The mean kyphotic angles after 12 months differed significantly, at $12.4^{\circ}$ and $13.6^{\circ}$ in the TFP and PVP groups, respectively $(p=0.013)$.

Five patients in the PVP group experienced adjacent compression fractures within 12 months, which differed signifi-

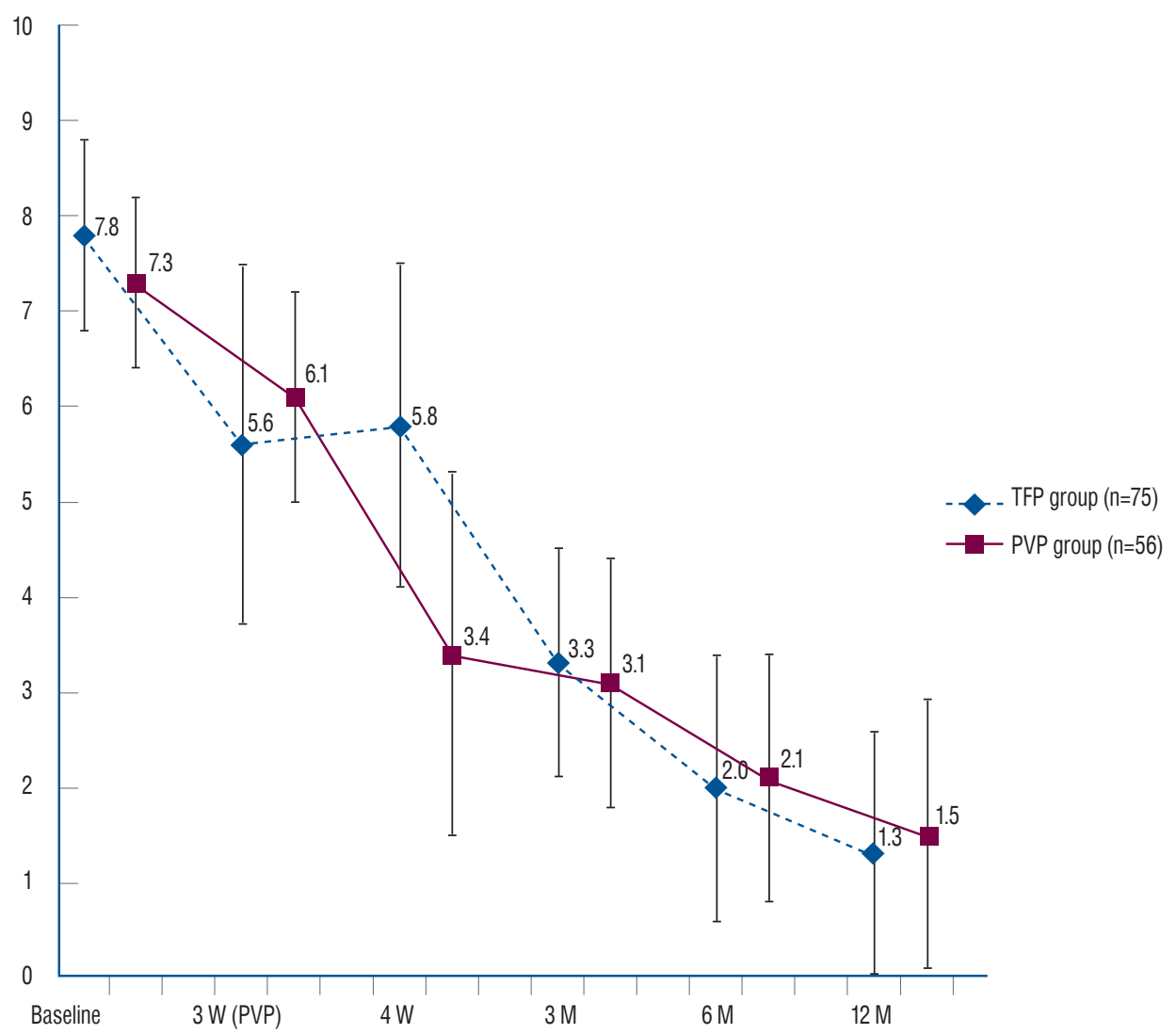

Fig. 4. Levels of pain over time in the percutaneous vertebroplasty (PVP) and transdermal fentanyl patch (TFP) groups. Pain reduction was greater in the PVP group than in the TFP group 1 week after the PVP procedure (4 weeks after osteoporotic vertebral compression fractures), but not subsequently. For each $p$-value, base line $=0.07,3$ weeks (the time at which PVP performed) $=0.355,4$ weeks $(1$ week after PVP) $=0.022,3$ months $=$ $0.601,6$ months $=0.626$, and 12 months $=0.484$. W : week(s), M : month(s). 
cantly from the TFP group, in which none were observed $(p=0.013)$ (Table 2). Adjacent compression fractures occurred within 1 month in two patients (L1 L2, T11 T12), within 3 months in two patients (T10 T9, T12 L1), and within 1 year in one patient (L1 L2) (Fig. 5).

\section{DISCUSSION}

Although PVP has become the preferred method of treatment, its relative benefits for OVCFs remain unclear ${ }^{7,10,16)}$. For example, a randomized controlled trial showed that pain relief and quality of life did not differ significantly between patients who underwent PVP and those who received conservative treatment $^{7}$. Moreover, a recent study found that most patients treated conservatively had favorable clinical results ${ }^{26)}$, and our study yielded similar results; although pain relief was greater in the PVP group 1 month after onset of OVCFs (1 week after PVP), the two groups subsequently showed similar pain relief.

Various imaging modalities have yielded varying results in assessing factors such as the compression ratio and kyphotic angle ${ }^{26)}$. In the present study, the compression ratio increased

Table 2. Baseline and 12-month compression fracture rates; 0 -, 6-, and 12-month vertebral body wedging angles and change from 0 to 12 months; and incidence of adjacent compression fracture in the two groups

\begin{tabular}{lccc}
\hline & TFP group $(\mathbf{n = 7 5 )}$ & PVP group $(\mathbf{n}=\mathbf{5 6})$ & \\
\hline Compression rate (\%) & & & \\
Baseline & $24.8 \pm 1.57$ & $32.9 \pm 2.17$ & 0.001 \\
12 months & $31.9 \pm 1.80$ & $28.1 \pm 2.27$ & 0.001 \\
Vertebral body wedging angle $\left(^{\circ}\right)$ & & & \\
Baseline & $13.2 \pm 3.4$ & $14.8 \pm 2.2$ & 0.002 \\
6 months & $13.1 \pm 3.3$ & $13.8 \pm 2.3$ & 0.064 \\
12 months & $12.4 \pm 3.2$ & $13.6 \pm 2.2$ & 0.013 \\
Development of adjacent vertebral fracture & & & $5(8.9)$ \\
$\quad$ Within 12 months & $0(0)$ & 0.013 \\
\hline
\end{tabular}

Values are presented as mean \pm standard deviation or number (\%). TFP : transdermal fentanyl patch, PVP : percutaneous vertebroplasty
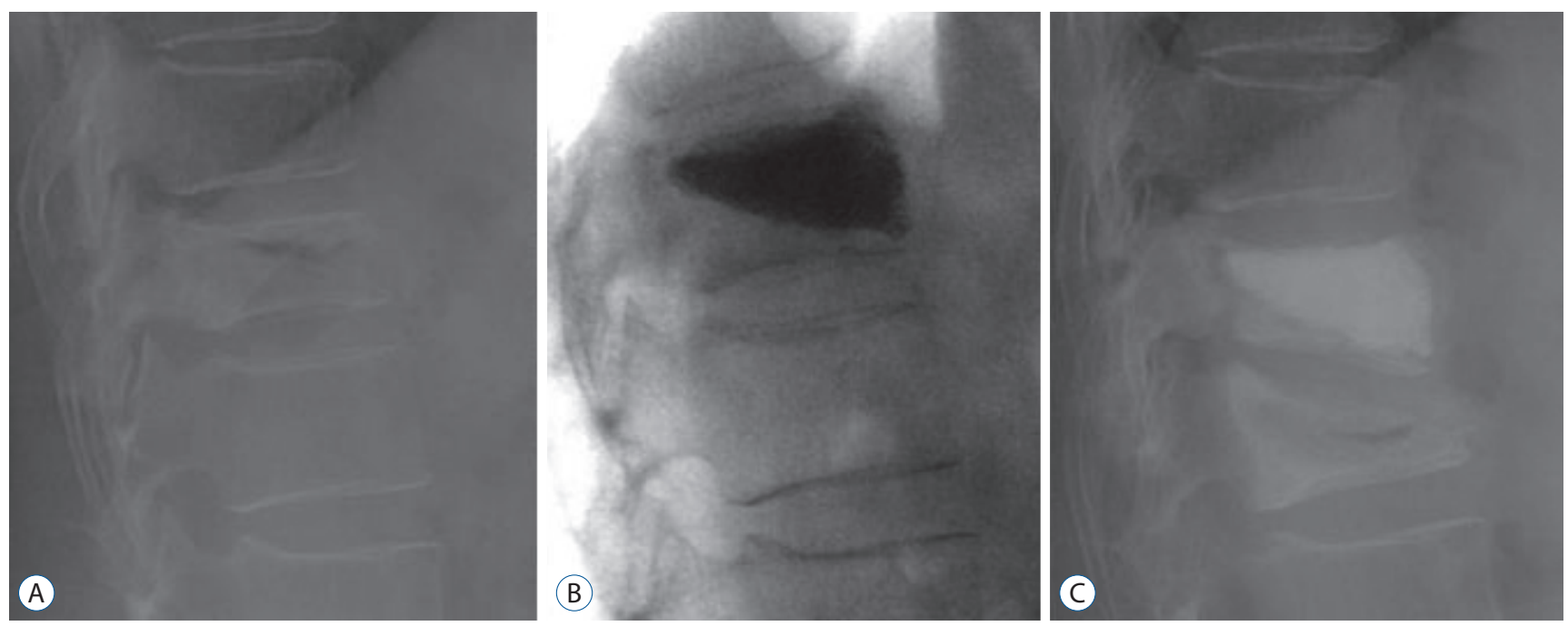

Fig. 5. X-rays of a representative patient with an adjacent compression fracture. A : X-ray showing a compression fracture at T11 in a 72 -year-old woman with a bone mineral density of -3.12 who presented with back pain after slipping. B:X-ray showing a decrease in the compression rate at T11 after postural reduction and percutaneous vertebroplasty (PVP) of the T11 vertebral body. C : Follow-up X-ray taken 1 month later because of pain without recurrent trauma, showing a new compression fracture at T12. Despite the adjacent compression fracture, the compression rate achieved by the previous PVP was well maintained. 
after 12 months in the TFP group but decreased after 12 months in the PVP group. Because PVP involves postural reduction, kyphosis reduction is maintained for at least 12 months ( $31.9 \%$ vs. $28.1 \%, p=0.001)$. The initially significantly worse compression rate in the PVP group might have been due to the study including only patients for whom the initial 3 weeks of conservative treatment failed $(24.8 \%$ vs. $32.9 \%$, $p=0.001$ ).

However, the kyphotic angle improved in both groups over time. Although the between-group difference in the change in the kyphotic angle was statistically significant, the actual difference was less than $1^{\circ}$, suggesting that this difference may not be clinically important and may have been due to measurement bias.

Several adverse effects have been associated with vertebral augmentation, including cement leakage and adjacent segment fractures ${ }^{5}$. The complications of cement leakage include neurologic deficits and pulmonary embolisms. Moreover, the incidence of new OVCFs after vertebroplasty has been reported to range from $8 \%$ to $52 \%{ }^{23)}$, with $41-69 \%$ of new vertebral compression fractures being immediately adjacent to the treated vertebra ${ }^{8,11,14,15,18,22)}$.

Five of our patients (8.9\%) who underwent PVP, but none in the TFP group, were diagnosed with adjacent compression fractures within 1 year and mostly within 3 months. Previous studies have also shown that most new OVCFs occur within 3 months after PVP ${ }^{8,11,14,15,18,22)}$.

Although age differed significantly in the present study (8.1 years, TFP group, 63.4 years old vs. PVP group, 71.5 years old), BMD was similar in the two groups $(p=0.751)$. Because the PVP group included only patients for whom the initial 3 weeks of conservative treatment failed, younger patients might have been excluded because of their better response to traditional conservative treatment. Collectively, the results indicate that conservative treatment might be more effective for OVCFs. In addition, we cannot exclude the possibility that more adjacent fractures occurred because of the older age in the PVP group rather than because of the direct stress effect of cement augmentation.

The reason for BMD scores being similar despite the significant difference in age between the two groups is believed to be the rapid decrease of BMD that generally occurs before the early 60s; a slow and gradual decrease occurs from the mid60 s to early 70s, resulting in little difference in BMD scores in our results ${ }^{3}$.

Generally, fentanyl patches have some of the same adverse effects as other opioids, mainly sedation, hypoventilation, nausea, vomiting, constipation, and urinary retention. In comparison with oral morphine, they cause fewer gastrointestinal adverse events. Because of the possibility of these side effects, we had to recommend that all patients be admitted to hospital and use individual fentanyl patches under careful monitoring. We could not administer fentanyl patches to 17 patients because of nausea and dizziness; however, no patients had a decrease in respiratory drive. Previously, we have shown the efficacy and safety of fentanyl patches for chronic pain ${ }^{17)}$. Fentanyl patches offer an alternative to oral morphine, and their effectiveness and tolerability have been demonstrated in several trials ${ }^{4}$. In addition to safety and experience, the most crucial reason to choose fentanyl patches instead of other oral opioids is the convenience of changing them every 3 days. Considering that $32.9 \%$ of the patients (56 out of 170 patients) underwent PVP rather than popular and traditional conservative treatment, but only $8.0 \%$ (six out of 75 patients) underwent PVP in the TFP group, our initial TFP application seemed to be more beneficial in reducing the conversion rate from conservative treatment to PVP if we carefully monitored the development of side effects.

This study had several limitations. First, this study had a retrospective design and was not a randomized control trial. Second, it measured only pain and radiologic factors including compression rate and kyphotic angle, but did not assess other functional outcomes and other sagittal radiological parameters. Finally, this study was non-homogeneous, in that the two groups of patients were treated by two independent surgeons.

\section{CONCLUSION}

We compared clinical and radiological outcomes between the TFP and PVP groups. The immediate pain reduction effect was superior in the PVP group, but the final clinical outcomes were similar. Although the PVP group exhibited more preserved segmental alignment than the TFP group did for 1 year, the development of adjacent fractures was significantly higher. Although conservative treatment using TFPs seems to be beneficial in reducing the conversion rate from conserva- 
tive treatment to PVP, the rate of the occurrence of side effects (in this study, 22.6\%, 17 out of 75 patients) should be carefully monitored.

\section{CONFLICTS OF INTEREST}

No potential conflict of interest relevant to this article was reported.

\section{INFORMED CONSENT}

Informed consent was obtained from all individual participants included in this study.

\section{AUTHOR CONTRIBUTIONS}

\author{
Conceptualization : JP \\ Data curation : YO, JK \\ Formal analysis : BL \\ Funding acquisition : $\mathrm{YO}$ \\ Methodology : BL \\ Project administration : JP \\ Visualization : SL \\ Writing - original draft : $\mathrm{YO}$ \\ Writing - review \& editing : JP
}

\section{References}

1. Anselmetti GC, Bernard J, Blattert T, Court C, Fagan D, Fransen $H$, et al. : Criteria for the appropriate treatment of osteoporotic vertebral compression fractures. Pain Physician 16 : E519-E530, 2013

2. Choi JH, Kang HD, Park JH, Gu BS, Jung SK, Oh SH : The efficacy of fentanyl transdermal patch as the first-line medicine for the conservative treatment of osteoporotic compression fracture. Korean J Neurotrauma 13 : 130-136, 2017

3. Cui R, Zhou L, Li Z, Li Q, Qi Z, Zhang J : Assessment risk of osteoporosis in Chinese people: relationship among body mass index, serum lipid profiles, blood glucose, and bone mineral density. Clin Interv Aging $11: 887-895,2016$

4. Grond S, Radbruch L, Lehmann KA : Clinical pharmacokinetics of transdermal opioids: focus on transdermal fentanyl. Clin Pharmacokinet $38: 59-89,2000$
5. Han S, Wan S, Ning L, Tong Y, Zhang J, Fan S: Percutaneous vertebroplasty versus balloon kyphoplasty for treatment of osteoporotic vertebral compression fracture: a meta-analysis of randomised and nonrandomised controlled trials. Int Orthop 35 : 1349-1358, 2011

6. Johnell 0 , Kanis JA : An estimate of the worldwide prevalence and disability associated with osteoporotic fractures. Osteoporos Int 17 : 1726-1733, 2006

7. Kallmes DF, Comstock BA, Heagerty PJ, Turner JA, Wilson DJ, Diamond TH, et al. : A randomized trial of vertebroplasty for osteoporotic spinal fractures. N Engl J Med 361 : 569-579, 2009

8. Kamano H, Hiwatashi A, Kobayashi N, Fuwa S, Takahashi O, Saida Y, et al. : New vertebral compression fractures after prophylactic vertebroplasty in osteoporotic patients. AJR Am J Roentgenol 197 : 451-456, 2011

9. Klazen CA, Lohle PN, de Vries J, Jansen FH, Tielbeek AV, Blonk MC, et al. : Vertebroplasty versus conservative treatment in acute osteoporotic vertebral compression fractures (Vertos II): an open-label randomised trial. Lancet 376 : 1085-1092, 2010

10. Lee HM, Park SY, Lee SH, Suh SW, Hong JY : Comparative analysis of clinical outcomes in patients with osteoporotic vertebral compression fractures (OVCFs): conservative treatment versus balloon kyphoplasty. Spine J 12 : 998-1005, 2012

11. Li HM, Zhang RJ, Gao H, Jia CY, Zhang JX, Dong FL, et al. : New vertebral fractures after osteoporotic vertebral compression fracture between balloon kyphoplasty and nonsurgical treatment PRISMA. Medicine (Baltimore) 97 : e12666, 2018

12. Lin H, Bao LH, Zhu XF, Qian C, Chen X, Han ZB : Analysis of recurrent fracture of a new vertebral body after percutaneous vertebroplasty in patients with osteoporosis. Orthop Surg 2 : 119-123, 2010

13. Lin WC, Cheng TT, Lee YC, Wang TN, Cheng YF, Lui CC, et al. : New vertebral osteoporotic compression fractures after percutaneous vertebroplasty: retrospective analysis of risk factors. J Vasc Interv Radiol 19(2 Pt 1) : 225-231, 2008

14. Movrin I: Adjacent level fracture after osteoporotic vertebral compression fracture: a nonrandomized prospective study comparing balloon kyphoplasty with conservative therapy. Wien Klin Wochenschr 124 : 304-311, 2012

15. Nieuwenhuijse MJ, Putter $H$, van Erkel AR, Dijkstra PD : New vertebral fractures after percutaneous vertebroplasty for painful osteoporotic vertebral compression fractures: a clustered analysis and the relevance of intradiskal cement leakage. Radiology 266 : 862-870, 2013

16. Papanastassiou ID, Filis A, Gerochristou MA, Vrionis FD : Controversial issues in kyphoplasty and vertebroplasty in osteoporotic vertebral fractures. Biomed Res Int 2014 : 934206, 2014

17. Park JH, Kim JH, Yun SC, Roh SW, Rhim SC, Kim CJ, et al. : Evaluation of efficacy and safety of fentanyl transdermal patch (Durogesic D-TRANS) in chronic pain. Acta Neurochir (Wien) 153 : 181-190, 2011

18. Pflugmacher R, Schroeder RJ, Klostermann CK : Incidence of adjacent vertebral fractures in patients treated with balloon kyphoplasty: two years' prospective follow-up. Acta Radiol 47 : 830-840, 2006

19. Rousing $\mathrm{R}$, Hansen $\mathrm{KL}$, Andersen MO, Jespersen SM, Thomsen $\mathrm{K}$, Lau- 
ritsen JM : Twelve-months follow-up in forty-nine patients with acute/ semiacute osteoporotic vertebral fractures treated conservatively or with percutaneous vertebroplasty: a clinical randomized study. Spine (Phila Pa 1976) 35 : 478-482, 2010

20. Rzewuska M, Ferreira M, McLachlan AJ, Machado GC, Maher CG : The efficacy of conservative treatment of osteoporotic compression fractures on acute pain relief: a systematic review with meta-analysis. Eur Spine J 24 : 702-714, 2015

21. Silverman SL: The clinical consequences of vertebral compression fracture. Bone 13 Suppl 2 : S27-S31, 1992

22. Uppin AA, Hirsch JA, Centenera LV, Pfiefer BA, Pazianos AG, Choi IS : Occurrence of new vertebral body fracture after percutaneous vertebroplasty in patients with osteoporosis. Radiology 226 : 119-124, 2003

23. Voormolen $M H$, Lohle PN, Juttmann JR, van der Graaf $Y$, Fransen $H$, Lampmann LE : The risk of new osteoporotic vertebral compression fractures in the year after percutaneous vertebroplasty. J Vasc Interv Radiol 17 : 71-76, 2006

24. Xie L, Zhao ZG, Zhang SJ, Hu YB : Percutaneous vertebroplasty versus conservative treatment for osteoporotic vertebral compression fractures: an updated meta-analysis of prospective randomized controlled trials. Int J Surg 47 : 25-32, 2017

25. Yang EZ, Xu JG, Huang GZ, Xiao WZ, Liu XK, Zeng BF, et al. : Percutaneous vertebroplasty versus conservative treatment in aged patients with acute osteoporotic vertebral compression fractures: a prospective randomized controlled clinical study. Spine (Phila Pa 1976) 41 : 653660,2016

26. Yi HJ, Jeong JH, Im SB, Lee JK : Percutaneous vertebroplasty versus conservative treatment for one level thoracolumbar osteoporotic compression fracture: results of an over 2-year follow-up. Pain Physician 19 : E743-E750, 2016 